\title{
A Rare Cause of Anterior Knee Pain in a Young Athlete and a Delayed Diagnosis: Osteoid Osteoma of the Patella
}

\author{
Eftychios Papagrigorakis $^{1}$, Ioannis S. Benetos ${ }^{2}$, Matthaios Bakalakos ${ }^{2}$, Meletis Rozis ${ }^{2}$, Spiros \\ Pneumaticos 3 \\ 1. Orthopaedics, KAT Trauma Hospital, Athens, GRC 2. Orthopaedics, University of Athens, KAT Trauma Hospital, \\ Athens, GRC 3. Orthopaedics, National and Kapodistrian University of Athens, KAT Hospital, Athens, GRC
}

Corresponding author: Eftychios Papagrigorakis, efpapagr@hotmail.com

\begin{abstract}
Intra-articular osteoid osteomas are a rare cause of articular pain. Their diagnosis can be challenging due to their non-specific clinical presentation and imaging characteristics. We present the case of a young soccer player with a 12-month history of anterior knee pain that was first attributed to Sinding Larsen Johansson syndrome and then to Hoffa's syndrome. A CT scan was performed that revealed the localization of an osteoid osteoma of the patella. The patient was successfully treated by percutaneous radiofrequency ablation under CT guidance without complications and returned to full sports activity. Although a rare entity, osteoid osteoma of the patella with its atypical clinical features could be included in the differential diagnosis of persistent anterior knee pain in the young adult. High clinical suspicion is necessary to avoid delay in diagnosis and irrelevant procedures for the patient.
\end{abstract}

Categories: Orthopedics

Keywords: osteoid osteoma, patella, anterior knee pain, athlete

\section{Introduction}

Osteoid osteoma (OO) is a benign bone lesion that constitutes $11 \%$ to $14 \%$ of all benign bone tumors and presents clinically with pain typically worsened at night, alleviated by nonsteroidal anti-inflammatory drugs (NSAIDs) or aspirin. The lesion is commonly found on the diaphysis or metaphysis of long bones, and its typical radiological appearance is a radiolucent zone surrounded by sclerotic bone (nidus) smaller than 1.5 $\mathrm{cm}$ in diameter [1].

Intra- and juxta-articular $\mathrm{OO}$ are a diagnostic challenge for the orthopedist due to their rare appearance (13\% of the lesions) and also their atypical clinical and radiological characteristics [2]. Misdiagnosis and delay till definitive treatment is a common problem, especially when the lesions arise in a subchondral location in the knee or the patellofemoral joint [3].

Received 12/09/2019

Review began 12/13/2019 Review ended 12/16/2019 Published 12/19/2019

\section{(c) Copyright 2019}

Papagrigorakis et al. This is an open access article distributed under the terms of the Creative Commons Attribution License CC-BY 3.0., which permits unrestricted use, distribution, and reproduction in any medium, provided the original author and source are credited.
We present the case of a patellar OO in a young soccer player that was treated by percutaneous ablation. The uncommon site in combination with the atypical clinical presentation caused a 13-month interval between the onset of the symptoms and final treatment.

The aim of this paper is to report this rare case of patellar OO presenting as anterior knee pain (AKP) along with a review of the literature. The difficulties a clinician faces, in his effort to diagnose a rare entity presenting with a vague knee symptomatology and thus focus on crucial points in the diagnosis of intra- and juxta-articular OO, should be highlighted.

\section{Case Presentation}

A 17-year-old adolescent soccer player presented with a 12-month history of left AKP. The pain was initially mild and poorly localized around the knee joint and had been attributed to a direct blow on the patella he had sustained during training. However, there was no relief with conservative treatment and physiotherapy and the pain progressively deteriorated over months. The pain was present at day and night and partially relieved with NSAIDs. An x-ray of the knee had been obtained with unremarkable findings (Figure 1). Two MRI scans had been performed with a six-month interval, both showing diffuse bone edema over the patella and mild effusion without any specific finding (Figure 2). Sinding Larsen Johansson syndrome was initially suspected, and sports participation was restricted. As the complaints became more intense, the patient stopped athletic activities and after the second MRI scan, Hoffa's syndrome was diagnosed. 


\section{Cureus}

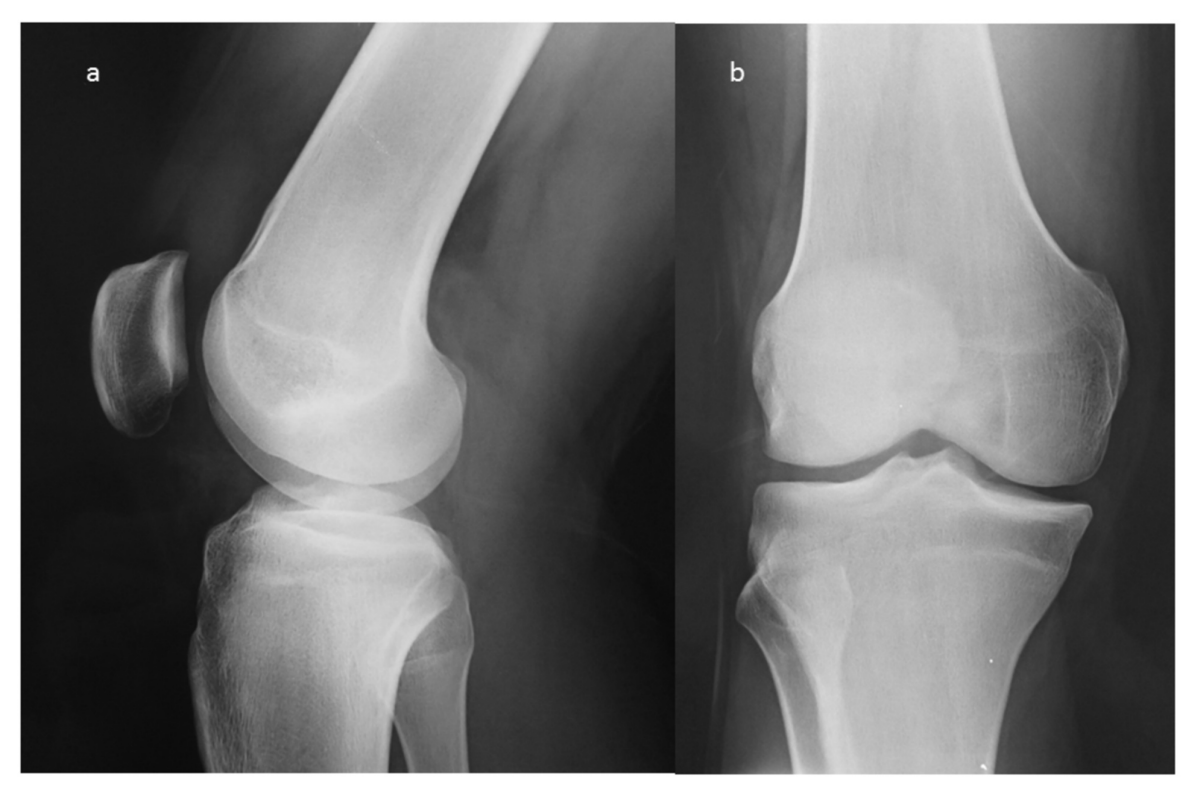

\section{FIGURE 1: Knee x-ray of the patient}

a. Sagittal view, b. Coronal view

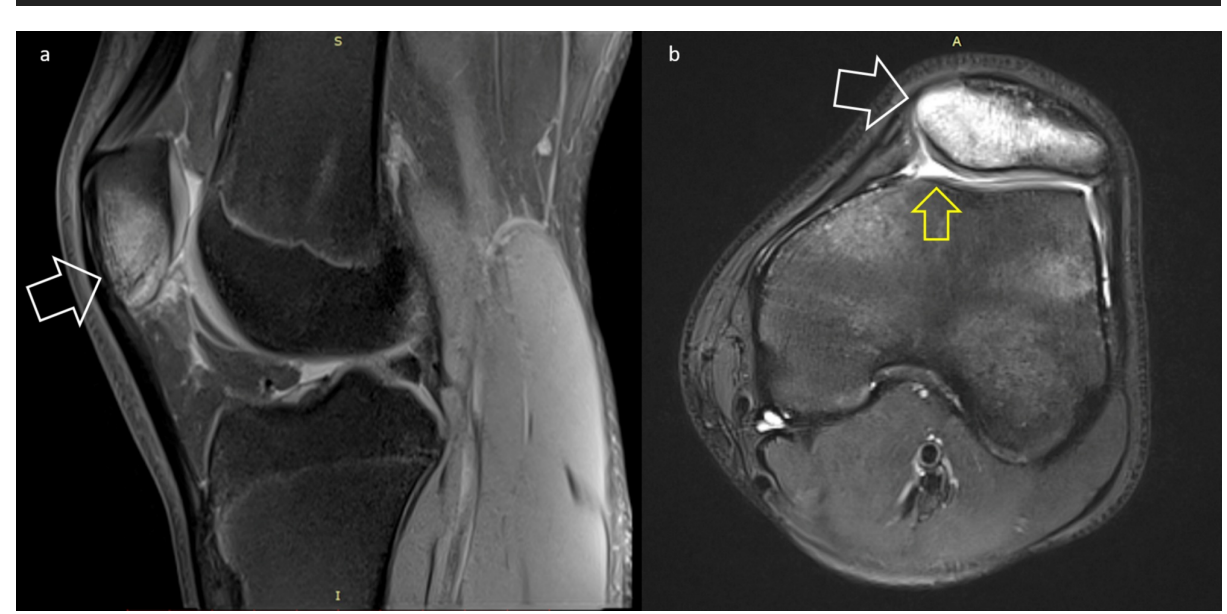

FIGURE 2: First MRI scan showing diffuse signal uptake of the patella and effusion

a. Sagittal view, b. Transverse view

White arrows: diffuse signal uptake, Yellow arrow: knee effusion

The pain gradually worsened over the next months, and the patient was referred to our institution for further treatment. At clinical examination, serious sensitivity was noted on the patella and patellofemoral joint without any redness or local temperature rise. Range of motion was limited due to intense pain, and was aggravated by patellar squeezing. Thigh atrophy was also obvious. Laboratory studies were unremarkable. A third MRI was performed showing diffuse signal uptake on the patella and joint effusion (Figure 3). 


\section{Cureus}

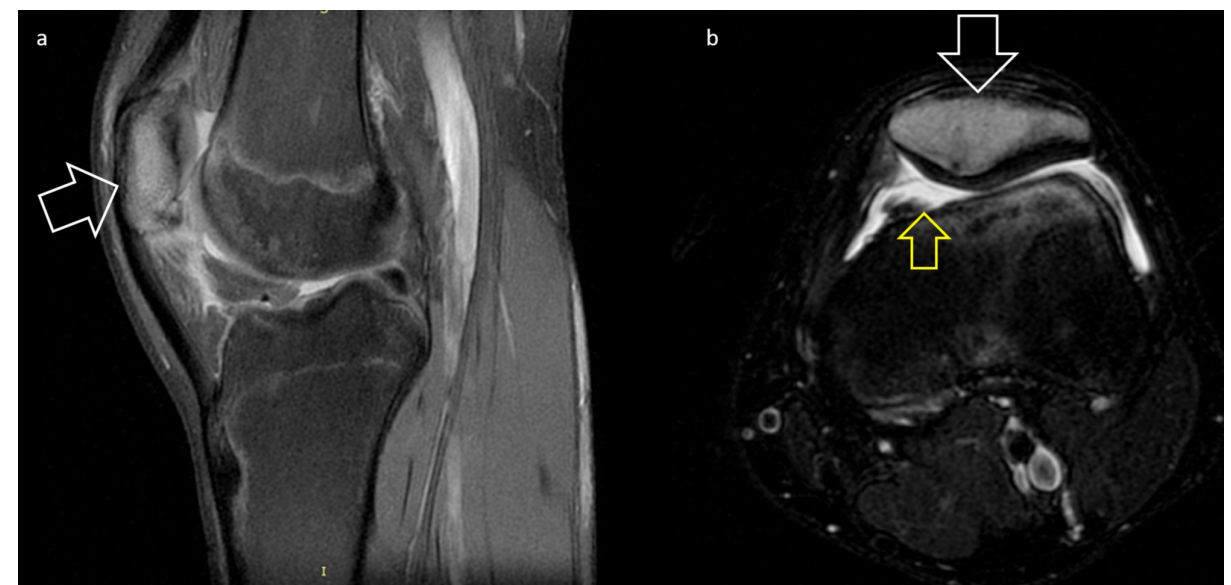

FIGURE 3: Third MRI scan, showing only non-specific findings, increased signal on $\mathrm{T} 2$ sequence, and mild effusion

a. Sagittal view, b. Transverse view

White arrows: increased signal on T2 sequences, Yellow arrow: knee effusion

T2: T2-weighted image

A diagnostic arthroscopy was scheduled. Before that a bone scan was performed, which showed intense focal uptake over the left patella (Figure 4). A CT scan followed revealing the classic nidus of an intra-articular patella OO, 12 months after the first symptoms (Figure 5). Because of the pathognomonic appearance of the nidus on the CT scan along with the results of the bone scan, the arthroscopy was cancelled and a CT-guided radiofrequency (RF) ablation was scheduled.

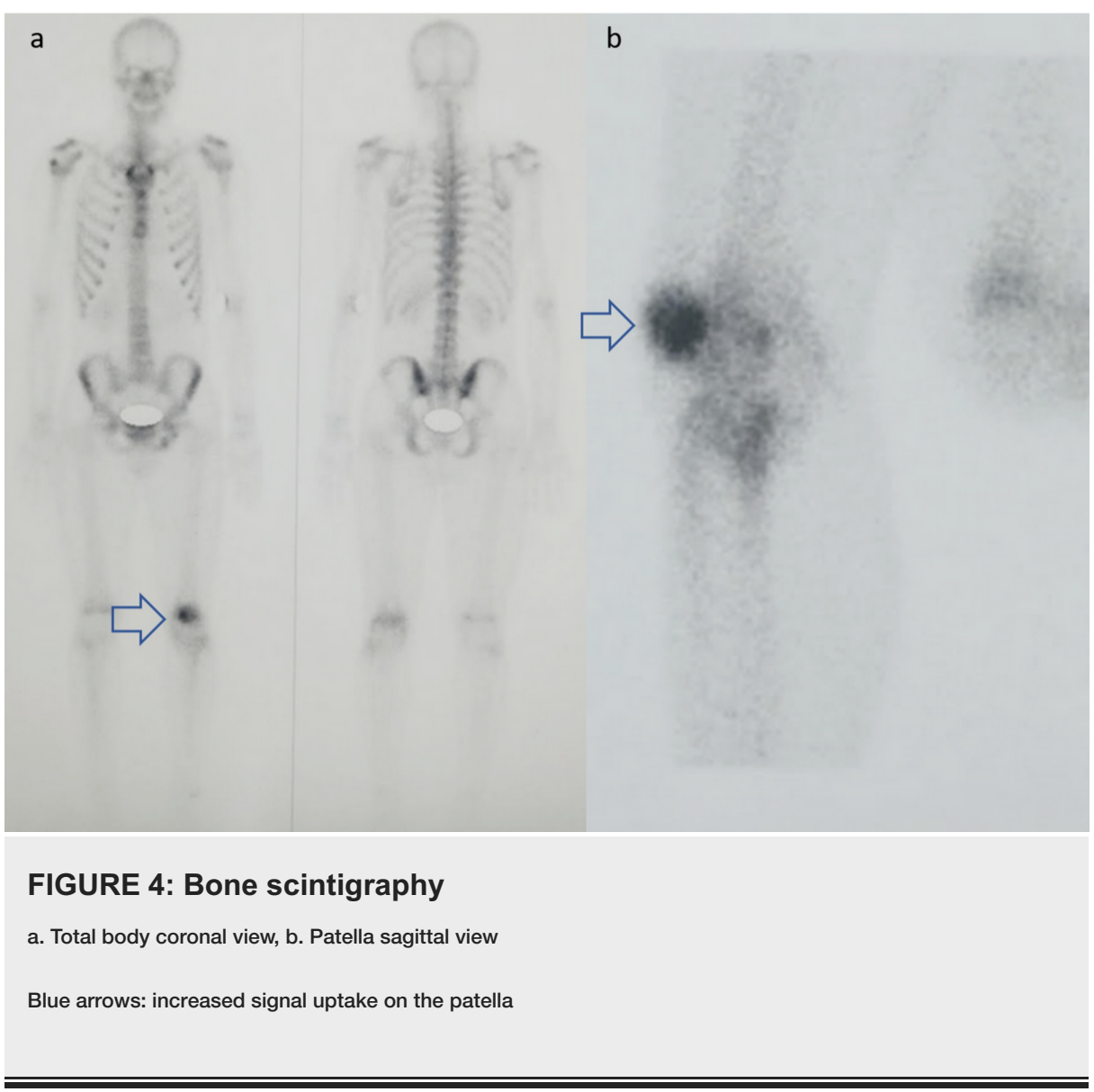




\section{Cureus}

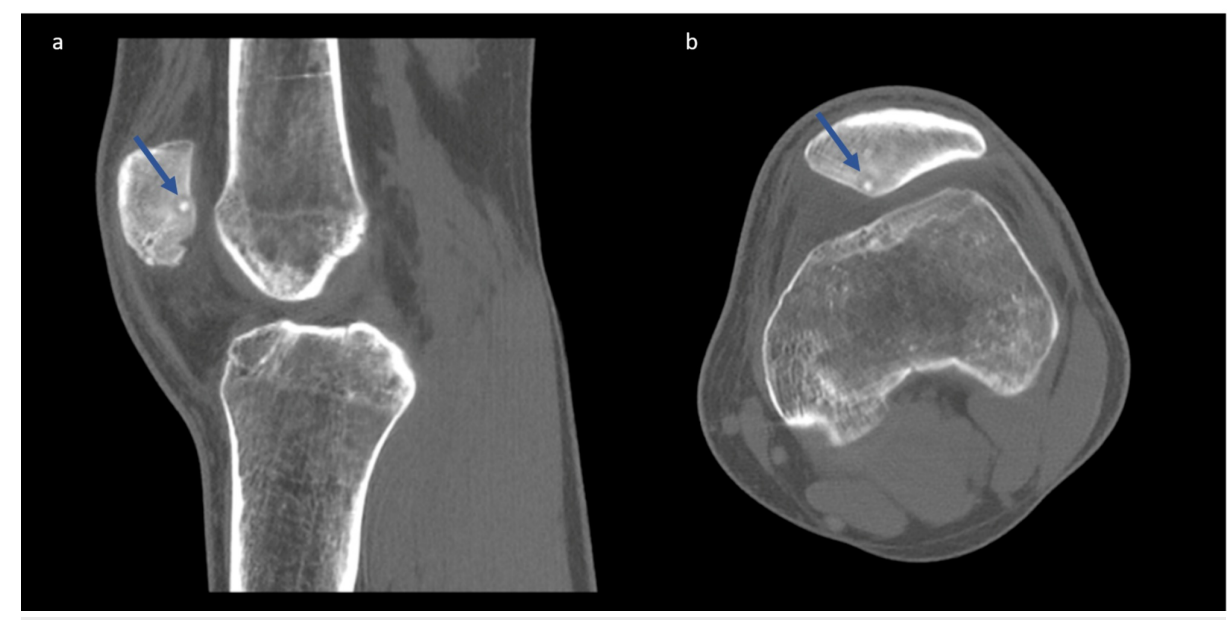

FIGURE 5: CT scan reveals the pathognomonic nidus appearance

Blue arrows: characteristic nidus on a subchondral location

The following month, a CT-guided RF ablation was performed. Prophylactic antibiotic treatment was administered one hour prior to the procedure. Under femoral block, without the use of a tourniquet, a small portal through the skin and the subcutaneous tissue was created with a no 11 scalpel on the anterior aspect of the patella. Through it, (avoiding any transarticular approach) a biopsy needle was transosseously inserted under CT guidance and a bony sample was obtained from the nidus. The tip of the RF electrode was precisely placed in the nidus (Figure 6), keeping a distance of $2 \mathrm{~mm}$ from the cartilage and RF ablation was performed.

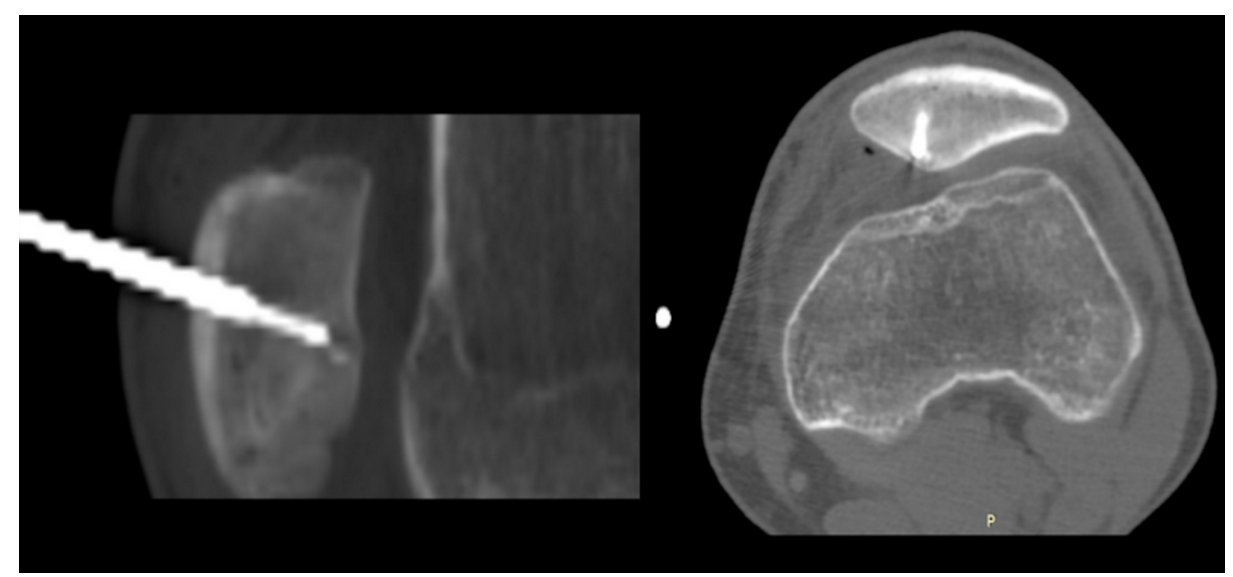

\section{FIGURE 6: CT-guided RF ablation}

The needle tip (white), as it approaches the nidus

RF: radiofrequency

Histological study confirmed the diagnosis of $\mathrm{OO}$, with the classic appearance of mineralized woven bone with regularly shaped nuclei containing little chromatin but abundant cytoplasm (Figure 7). 


\section{Cureus}

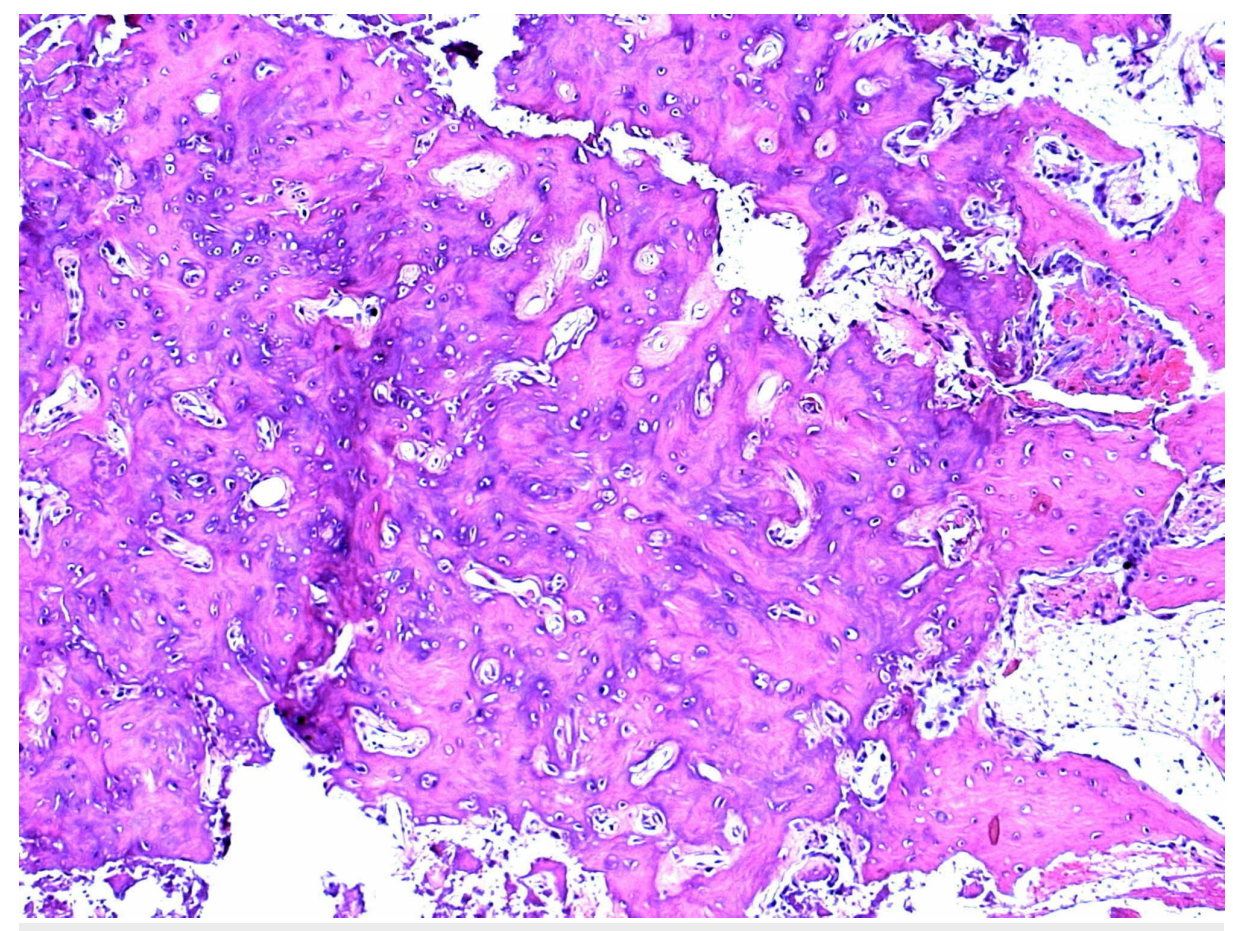

FIGURE 7: The classic appearance of the nidus of the osteoid osteoma composed of bony trabeculae, separated by vascular fibrous connective tissue

The patient remained hospitalized overnight and was discharged the day after the procedure with a mild discomfort on the ablation site, with full range of motion. He was advised to use crutches for the first two weeks and then proceed to full weight bearing. He returned to full sports activity one month after the procedure without any complaints. On his regular follow-up 1, 6, and 24 months following the ablation, he remained completely free of pain and satisfied with his sports performance.

\section{Discussion}

The unique imaging features of intra-articular OO combined with their common clinical presentation make them a diagnostic challenge for the clinician. Kattapuram et al. suggested that they should be considered separate clinical entities because of the difficulties encountered in their recognition [4]. Especially around the knee joint the diagnosis can be delayed for many months [3]. In our case, the interval between the first symptoms and diagnosis was 12 months.

The first arduousness is that $\mathrm{OO}$ are a rare cause of joint pain. Approximately $5.2 \%$ to $13 \%$ of the $\mathrm{OO}$ appear in a juxta- or intra-articular position [5,6]. Hip joint is the most commonly affected area, followed by the ankle, elbow, wrist, and knee $[2,6]$. Patella is a rare site for 00 , and only a few cases have been reported in the literature [7-13].

The most common symptom when an $\mathrm{OO}$ arises around the knee joint is AKP. However, AKP is one of the most frequently met musculoskeletal disorders. Each year many young athletes seen in primary care setting complain of some degree of knee pain, which is usually attributed to chondromalacia patella, patellar tendinitis, mediopatellar plica syndrome, Hoffa's syndrome, patellofemoral malalignment, osteochondritis dissecans, meniscal tears, or ligamentous injuries [14]. The pain encountered from an $\mathrm{OO}$ is the result of the high prostaglandin levels produced within the nidus [15]. The transmission of these prostaglandins from the nidus to synovium causes lymphofollicular synovitis, resembling histologically rheumatoid arthritis and clinically monoarthritis of infectious, degenerative, or rheumatologic origin [16]. In some cases of intraarticular $\mathrm{OO}$ as the one presented here, relief with salicylates or NSAIDs may be partial and typical nocturnal pain absent. The diffuse pain due to synovitis and the lesion itself accompanied by non-specific symptoms as muscle atrophy or muscle spasm around the joint, limited range of motion, joint effusion and swelling, gait and postural disturbances may be misleading for the clinician [2].

Imaging features of the intra-articular $\mathrm{OO}$ differ from the characteristics of the extra-articular $\mathrm{OO}$. $\mathrm{X}$ rays are not diagnostic in $80 \%$ of the cases as at the time of the first symptoms no nidus or periosteal reaction is usually present $[3,4,17]$. On the other hand, intracapsular periosteum seems less able to produce thick new bone because of the absence of cambium layer and thus periosteal reaction surrounding the nidus may be 
minimal or absent when the lesion is located within $1 \mathrm{~cm}$ from the articular surface [2,4]. Another common radiological finding is juxta-articular osteopenia or osteoarthritic changes [2,18]. In scintigraphy the typical double density sign is usually absent showing diffuse uptake and generalized involvement of the joint (as in our case, Figure 4) decreasing the specificity of the examination [6,16-18].

MRI is often requested as first-line imaging when dealing with knee symptoms. In our case, three MRI scans were performed in 12 months, none of which revealed the lesion, showing diffuse bone edema over the patella and joint effusion. In the literature, it is reported that approximately $21 \%$ of intra-articular niduses are not identified and a further $29 \%$ poorly identified, on initial MRI [6]. The increased signal in some cases may even "transform" a benign lesion into an aggressive lesion and the picture can be mistaken by the radiologist as a sarcoma of bone [18]. CT scans are still considered the gold standard for detecting and diagnosing $\mathrm{OO}$ both in adults and pediatric population [18]. For our patient, CT proved more accurate than MRI in detection of the OO, giving us the characteristic nidus demonstration (Figure 5).

Newer techniques have shown promising results in the imaging of intra-articular OO. Especially the use of single photon emission computed tomography/CT and MRI gadolinium-enhanced imaging have proved to be efficient in detection of the nidus in intra-articular lesions, which may have been the reason for a relatively early diagnosis $[5,16]$. According to Szendroi et al. a careful diagnostic approach with the simultaneous use of several kinds of imaging makes it possible to confirm the diagnosis in $80 \%$ of cases of intra-articular OO before planning treatment, especially in the knee [17].

The average delay for diagnosis of intra-articular OO has been reported by Szendroi et al. at 26.6 months and by Rolvien et al. at 20.7 months [5,17]. For patellar OO, except for a case in a 13-year-old girl where diagnosis came six months after the first symptoms, the delay till diagnosis ranges from 12 months (in our case) to three years $[9,12]$.

The aforementioned imaging difficulties accompanied by the persistent non-specific symptoms of the patellar OO can result in misdiagnosis, irrelevant surgical procedures, and great discomfort for the patient. In our case, three MRI scans and physiotherapies were performed and an arthroscopy was scheduled. Our patient presented with an ill-defined AKP that was first attributed to a mild injury during training and after that to Sinding Larsen Johansson and Hoffa's syndrome before final diagnosis. Other common misdiagnosis for patella $\mathrm{OO}$ across the literature includes chondromalacia patella in three cases: patellofemoral malalignment, osteochondritis dissecans, meniscal tear, and quadricept tendinitis [7-9,12,13]. Half of the cases underwent at least one diagnostic arthroscopy before final diagnosis $[8,10,13]$. Bavaneh et al. described a patellar OO case where the patient had three MRI scans, one diagnostic arthroscopy followed by synovial biopsy and a psychiatric consultation before the CT scan that revealed the nidus, and final treatment with open resection and mosaicplasty [13]. Cohen et al. reported a patella $\mathrm{OO}$ case where two diagnostic arthroscopies and a femoral biopsy were performed before diagnosis and open resection [10].

Although the standard of care for the treatment of $\mathrm{OO}$ is image-guided RF ablation, in subchondral lesions, as in our case, cartilage degeneration is a possible complication and needs attention, especially in weightbearing joints $[5,19]$. A distance of $1 \mathrm{~mm}$ or more from the cartilage is considered safe, and articular cartilage appears to be relatively tolerant to thermal injury induced by short-term heating [20]. For most patellar OO, open resection was the chosen treatment, while Bavaneh et al. applied mosaicplasty technique after open resection for the cartilage defect $[7,10,13]$. On the other hand, Franceschi et al. described a CT-guided arthroscopic resection of a subchondral patellar $\mathrm{OO}$ and Chillemi et al. described a percutaneous CT-guided drilling of the nidus with good results $[11,12]$. Our patient was successfully treated with the method of RF ablation under CT guidance (Figure 6 ) without any cartilage complications as implied by the complete resolution of the symptoms and absence of complaints during follow-up.

\section{Conclusions}

Although a rare entity, OO of the patella with its atypical clinical features should be included in the differential diagnosis of persistent AKP in the young adult. Especially in adolescents presenting with longstanding non-specific complaints around the knee joint with non-conclusive MRI findings, CT scan seems a reasonable step in the diagnostic procedure. High clinical suspicion for this rare lesion is necessary for the orthopedist to avoid delay in diagnosis and irrelevant procedures for the patient. Percutaneous CT ablation seems a safe technique for the treatment of patellar OO, although attention is needed to avoid any cartilage damage.

\section{Additional Information \\ Disclosures}

Human subjects: Consent was obtained by all participants in this study. Conflicts of interest: In compliance with the ICMJE uniform disclosure form, all authors declare the following: Payment/services info: All authors have declared that no financial support was received from any organization for the submitted work. Financial relationships: All authors have declared that they have no financial relationships at present or within the previous three years with any organizations that might have an 
interest in the submitted work. Other relationships: All authors have declared that there are no other relationships or activities that could appear to have influenced the submitted work.

\section{References}

1. Orth P, Kohn D: Diagnostics and treatment of osteoid osteoma . Orthopade. 2017, 46:510-521. 10.1007/s00132-017-3428-0

2. Ciftdemir M, Tuncel SA, Usta U: Atypical osteoid osteomas. Eur J Orthop Surg Traumatol. 2015, 25:17-27. 10.1007/s00590-013-1291-1

3. Georgoulis AD, Papageorgiou CD, Moebius UG, Rossis J, Papadonikolakis A, Soucacos PN: The diagnostic dilemma created by osteoid osteoma that presents as knee pain. Arthroscopy. 2002, 18:32-37. 10.1053/jars.2002.30010

4. Kattapuram SV, Kushner DC, Phillips WC, Rosenthal DI: Osteoid osteoma: an unusual cause of articular pain. Radiology. 1983, 147:383-387. 10.1148/radiology.147.2.6836118

5. Rolvien T, Zustin J, Mussawy H, Schmidt T, Pogoda P, Ueblacker P: Intra-articular osteoid osteoma as a differential diagnosis of diffuse mono-articular joint pain. BMC Musculoskelet Disord. 2016, 17:455. 10.1186/s12891-016-1313-3

6. Allen SD, Saifuddin A: Imaging of intra-articular osteoid osteoma . Clin Radiol. 2003, 58:845-852. 10.1016/s0009-9260(03)00213-7

7. Vallianatos PG, Tilentzoglou AC, Seitaridis SV, Mahera HJ: Osteoid osteoma of the patella: a case report . Knee Surg Sports Traumatol Arthrosc. 2006, 14:161-164. 10.1007/s00167-005-0653-6

8. Koós Z, Than P: Rare localization of osteoid osteoma in the patella . Pediatr Radiol. 2005, 35:929-930. 10.1007/s00247-005-1476-9

9. Altinel L, Degirmenci B, Kose KC, Sahin O: Percutaneous resection of a patellar osteoid osteoma using a cannulated skin punch biopsy needle. Arch Orthop Trauma Surg. 2007, 127:299-302. 10.1007/s00402-0060108-7

10. Cohen I, Aner A, Rzetelny V: Osteoid osteoma of the patella . Harefuah. 1997, 132:463-465.

11. Franceschi F, Longo UG, Ruzzini L, et al.: En-bloc retrograde resection of an osteoid osteoma of the patella using computed tomography under arthroscopic control. J Knee Surg. 2008, 21:136-40. 10.1055/s-00301247808

12. Chillemi C, Franceschini V, D'Erme M, Ippolito G, Farsetti P: Patellar osteoid osteoma as a cause of anterior knee pain in adolescents: a case report and literature review. Case Rep Med. 2013, 2013:746472. $10.1155 / 2013 / 746472$

13. Bavaneh MK, Kiyak G, Balikci T: A review of literature: mosaicoplasty as an alternative treatment for resection of patellar osteoid osteoma and cartilage reconstruction. J Orthop. 2018, 15:768-771. 10.1016/j.jor.2018.05.036

14. Samim M, Smitaman E, Lawrence D, Moukaddam H: MRI of anterior knee pain. Skeletal Radiol. 2014, 43:875-893. 10.1007/s00256-014-1816-7

15. Kawaguchi Y, Sato C, Hasegawa T, Oka S, Kuwahara H, Norimatsu H: Intraarticular osteoid osteoma associated with synovitis: a possible role of cyclooxygenase-2 expression by osteoblasts in the nidus. Mod Pathol. 2000, 13:1086-1091. 10.1038/modpathol.3880202

16. Jordan RW, Koç T, Chapman AW, Taylor HP: Osteoid osteoma of the foot and ankle: a systematic review . Foot Ankle Surg. 2015, 21:228-234. 10.1016/j.fas.2015.04.005

17. Szendroi M, Kollo K, Antal I, Lakatos J, Szoke G: Intraarticular osteoid osteoma: clinical features, imaging results, and comparison with extra articular localization. J Rheumatol. 2004, 31:957-964.

18. Pikoulas C, Mantzikopoulos G, Thanos L, Passomenos D, Dalamarinis C, Glampedaki-Dagianta K: Unusually located osteoid osteomas. Eur J Radiol. 1995, 20:120-125. 10.1016/0720-048x(95)00636-5

19. Bosschaert PP, Deprez FC: Acetabular osteoid osteoma treated by percutaneous radiofrequency ablation: delayed articular cartilage damage. JBR-BTR. 2010, 93:204-206. 10.5334/jbr-btr.292

20. Eggel Y, Theumann N, Luthi F: Intraarticular osteoid osteoma of the knee: clinical and therapeutical particularities. Joint Bone Spine. 2007, 74:379-381. 10.1016/j.jbspin.2006.10.007 